\title{
Salud mental de universitarios víctimas del conflicto armado: una aproximación desde el método mixto
}

\author{
Drs. Yolima Alarcón-Vásquez ${ }^{1}$, Marly Johana Bahamón², Mabel Mercado Peñaloza³, Eugenio \\ Saavedra Guajardo ${ }^{4}$, Orlando González-Gutiérrez ${ }^{5}$
}

\begin{abstract}
RESUMEN
Introducción: La condición de víctima del conflicto armado supone la existencia de una serie de alteraciones en el desarrollo vital del sujeto que impactan la salud mental. Objetivo: Analizarfactores de riesgo y protección vinculados a la salud mental de víctimas del conflicto armado colombiano. Materiales y Método: Enfoque multimétodo y diseño concurrente en el cual se aplicó las escalas de: depresión de Zung, autoestima de Rosenberg, orientación vital $L O T-R$, estrés percibido, satisfacción con la vida de Diener, el inventario de ideación positiva y negativa
\end{abstract}

\section{DOI: https://doi.org/10.47307/GMC.2020.128.3.16}

Yolima Alarcón-Vásquez¹,yalarcon1@unisimonbolivar.edu.co , https://orcid.org/0000-0001-6357-8908.

Marly Johana Bahamón ${ }^{2}$, mbahamon@unisimonbolivar.edu.co, https://orcid.org/0000-0003-2528-994X

Mabel Mercado Peñaloza ${ }^{3}$, mmercado17@unisimonbolivar.edu. co, https://orcid.org/0000-0001-6802-2271,

Eugenio Saavedra Guajardo ${ }^{4}$, eugeniosaavedra@hotmail.com, http://orcid.org/0000-0003-2843-9739

Orlando González-Gutiérrez ${ }^{5}$; ogonzalez@unisimonbolivar.edu. co, Orcid: https://orcid.org/0000-0002-1123-7412

${ }^{1}$ Universidad Simón Bolívar, Facultad de Ciencias Jurídicas y Sociales, Barranquilla, Colombia.

${ }^{2}$ Universidad Simón Bolívar, Facultad de Ciencias Jurídicas y Sociales, Barranquilla, Colombia.

${ }^{3}$ Universidad Simón Bolívar, Programa Institucional de Excelencia Académica PIEA, Barranquilla, Colombia.

${ }^{4}$ Universidad Católica del Maule, Programa de Psicología, Chile.

${ }^{5}$ Universidad Simón Bolívar, Facultad de Ciencias Jurídicas y Sociales, Barranquilla, Cúcuta

Correspondencia: Yolima Alarcón-Vásquez. Facultad de Ciencias Jurídicas y Sociales, Universidad Simón Bolívar. Carrera 59 No 59-65. Teléfono: +57 53444333 Extensión 139. Barranquilla, Colombia. Correo electrónico: yalarcon1@ unisimonbolivar.edu.co.
PANSI, de resiliencia SV-RES y una guía de entrevista. Participaron 25 universitarios registrados ante la unidad de víctimas de Colombia. Resultados: Los datos sugieren un menoscabo en la salud mental de los participantes, representado en sintomatología depresiva, altos niveles de estrés y de ideación vinculada al suicidio y bajos puntajes en los factores de resiliencia. Asímismo, bajos niveles de autoestima y optimismo. Las fortalezas se ubicaron en la presencia pensamientos de protección frente al suicidio, tales como el sentido de control, la autoconfianza, las expectativas positivas, la alegría y la satisfacción con la vida. Conclusiones: Las instituciones de educación superior deben considerar la formulación de programas que promuevan el bienestar psicológico y la resiliencia en estos estudiantes.

Palabras clave: Conflicto armado, salud mental, víctimas, método mixto.

\section{SUMMARY}

Introduction: The condition of being a victim in the context of an armed conflict supposes the existence of a series of developmental disorders that might have an impact on mental health. Objective: Analyze risk and protection factors regarding the mental health of victims of the Colombian armed conflict. Material and Methods: Multimethod approach and concurrent design in which the Zung depression scale, the Rosenberg self-esteem scale, the LOT-R vital orientation scale, the perceived stress scale, the Diener life satisfaction scale, the PANSI positive and negative ideation inventory, the $S V$-RES resilience scale, and an interview guide. Twenty-five Colombian university students registered as victims participated in this study. 
Results: The data suggest a reduction in the mental health of the participants, represented in depressive symptomatology (although not clinical), high levels of stress, and the presence of ideation linked to suicide and low scores in resilience factors. Also, low levels of self-esteem and optimism. The strengths were only locatedinthe presence of thoughts of protection against suicide, such as the sense of control, self-confidence, positive expectations, joy, and satisfaction with life. Conclusions: That higher education institution that receives these students contemplates the formulation of programs that promote psychological well-being and resilience among this population.

Key words: Armed conflict, mental health, victims, mixed method.

\section{INTRODUCCIÓN}

El concepto de salud mental ha evolucionado a lo largo de los últimos años y pasó de ser considerada la ausencia de enfermedad mental para asumirse desde una perspectiva más integral, siendo considerada como aquel estado de bienestar en el cual una persona tiene la habilidad para desempeñarse adecuadamente en diferentes esferas de su vida, enfrentar las adversidades, los problemas y el estrés de la vida cotidiana y poder contribuir a su entorno $\operatorname{social}^{\&}(1-4)$. Lo anterior ha permitido comprender de mejor manera la complejidad de aquellos factores que afectan el bienestar de un individuo, la perspectiva considerada una "visión positiva" de la salud mental, ha logrado generar cambios sustanciales en las políticas de salud pública en todos los países (5).

En relación con la salud mental surge una gran problemática que ha venido afectando a la población colombiana y tiene que ver con la situación del conflicto armado en el que ha vivido nuestro país, ya que las personas que enfrentan situaciones violentas están propensas a sufrir daños psicológicos a corto, mediano y largo plazo, lo que evidentemente afecta la calidad de vida de esta población (6). Algunos estudios han demostrado que los individuos que han sufrido situaciones traumáticas tienen riesgo significativo de sufrir síntomas postraumáticos, padecer trastornos mentales; y desarrollar problemas en su vida familiar, personal y social; lo cual permite que su salud mental se vea afectada (6-8).
Es un hecho que la salud mental de gran parte de la población en Colombia se encuentra afectada, ya que así los demuestran los resultados de la Encuesta de Salud Mental (9) que se realizó en el 2015, en especial en la población víctima de conflicto armado hay una alta frecuencia de síntomas emocionales o trastornos mentales $(9,10,11-13)$.

De la misma manera ciertos estudios que reportan las implicaciones o secuelas psicológicas en combatientes y población civil expuesta a conflictos bélicos o similares que impliquen violencia. Algunas de las cifras exponen que hasta un $30 \%$ de las personas expuestas a violencia padecen síndrome de estrés postraumático y depresión, incluso se ha reportado un $100 \%$ en poblaciones con una afectación muy significativa $(14,15)$.

Por su parte autores como Campo-Arias y Herazo (10) consideran que en Colombia aquellas personas que han vivido en medio de la violencia y conflicto aumentan su problemática de su salud mental al verse sometidos a estigmas y discriminaciones. Así mismo, estas acciones violentas a las que fueron sometidas algunas poblaciones han generado un daño en el campo social, familiare incluso en la organización social y política en las que se encuentran incluidas las víctimas (8).

Investigaciones como las de Bell y col.(17) con población adulta víctima del conflicto armado, reportan comorbilidad entre trastorno de estrés postraumático (TEPT), ansiedad y depresión. Además, Londoño y col. (18) reportan una prevalencia de síntomas de ansiedad entre el $25,7 \%$ al $32,5 \%$, de trastorno de somatización entre $61 \%$ y $73,8 \%$, abuso de alcohol $(23,8 \%$ a $38,1 \%$ ) y trastornos de la alimentación entre $4,7 \%$ y $11,9 \%$.

Precisamente y con el fin de reevaluar las políticas que el Estado Colombiano había venido planteando para atender a la población víctima del conflicto se promulga la Ley 1448 o Ley de víctimas y restitución de tierras. En esta se establece que la atención a las víctimas incluye el acompañamiento psicosocial con el fin de que se logre la restitución real de los derechos (20). Para lograr esto dicha Ley estableció la creación del Programa De Atención Psicosocial y Salud Integral A Víctimas (PAPSIVI) en el cual se 
contempla claramente que las víctimas deben recibir una atención psicosocial de calidad a través de un equipo interdisciplinario.

Para Moreno y Díaz $(21,19)$ este enfoque finalmente reconoce la capacidad del individuo para ver el sufrimiento de manera distinta y no como una patología y está soportado por el hecho de que dicho sufrimiento es producto de los hechos violentos vividos, por lo tanto, el resultado del diagnóstico no debería ser considerado solo bajo criterios estandarizados. Frente a toda la situación antes mencionadas, surge un tema y tiene que ver con las oportunidades que este grupo social pueda mejorar su calidad de vida a través de la educación, pues ésta permite que las personas mejoren su calidad de vida $(22,23)$.

\section{MATERIALES Y MÉTODOS}

Se realizó un estudio mixto con diseño concurrente en el cual se aplicaron ambos métodos de manera simultánea. Los datos fueron recabados en el mismo espacio de tiempo para efectuar metainferencias a partir de los datos cuantitativos y cualitativos (24).

Mediante muestreo por conveniencia se incluyeron 25 jóvenes que se encontraban realizando estudios universitarios en una institución de educación superior y se identificaron plenamente en calidad de víctimas desde su registro ante la unidad de víctimas de Colombia. Se convocó a todos los estudiantes que se habían identificado previamente como víctimas y que contaban con el registro oficial y se trabajó con aquellos que asistieron voluntariamente. Los criterios de inclusión fueron a) estudiante universitario, b) registro ante la unidad de víctimas de Colombia, c) firmar el consentimiento informado. El rango de edad se ubicó entre 16 y 28 años con $(20,40 \pm 3,17), 19$ mujeres y 6 hombres, pertenecientes a estratos socioeconómicos 1,2 y 3 .

Se administró la Escala de depresión de Zung (25) se basa en aspectos afectivos, fisiológicos y psicológicos que caracterizan la depresión, está conformado por 20 reactivos con respuestas que varían entre 1 y 4 . Del total de los ítems 9 se encuentran redactados de forma negativa $(2,5,6,11,12,14,16,17$,
18 y 20). En Colombia, Lezama (26) identificó alfa de Cronbach de $r=0,548$. Los puntos de corte son: 20-35: depresión ausente, 36-51: depresión subclínica y variantes normales, 5267: depresión media-severa, 68-80: depresión grave. Además se aplicó la Escala de autoestima de Rosenberg (28), versión que cuenta con 10 ítems de los cuales cinco valoran la autoestima positiva (ítems $1,3,5,7$ y 9) y cinco la autoestima negativa (ítems $2,4,6,8$ y 10), con opciones de respuesta tipo Likert entre 1 y 4 . En Colombia fue validada por Ceballos y col. (27) quiénes reportan índices de confiabilidad de $\mathrm{r}=0,75$ para autoestima positiva y $\mathrm{r}=0,53$ para autoestima negativa. La Escala de Orientación vital LOT-R, Test de auto-reporte que evalúa el optimismo disposicional (28). Consta de diez ítems, tres afirmaciones sobre el optimismo (ítems 1,4 y 10), tres sobre el pesimismo (ítems 3,7 y 9) y cuatro ítems distractores $(2,5,6$ y 8$)$. Se responde en una escala de 1 a 5 su consistencia interna (alfa de Cronbach entre $r=0,70$ y 0,80 ) según Scheier y col. (28) y validado en Colombia por Velasco y col. (29).

También se aplicó la Escala de estrés percibido (30). En su versión corta consta de 10 ítems y cuenta con un patrón de respuesta que oscilaentre $0-4$. Los puntos $4,5,7, y$ se califican de manera inversa. Las preguntas miden el grado en que, durante el último mes, las personas se han sentido molestas o preocupadas o, por el contrario, se han sentido seguras de su capacidad para controlar sus problemas personales. Para su puntuación el estudio se ha basado en los hallazgos de Campo-Arias y col. (10), quienes refieren que esta escala presenta una estructura bifactorial, distrés general (ítems 1,2,3,6,9,10) con un $\mathrm{r}=0,83$ y capacidad de afrontamiento (ítems $4,5,7,8$ ) con un $\mathrm{r}=0,82$. La Escala de satisfacción con la vida Escala de Satisfacción con la Vida de Diener (31). Se compone de cinco ítems que evalúan la satisfacción con la vida mediante el juicio global que la persona hace. En este estudio se utilizó la traducción al castellano de Atienza y col. (32) con opciones de respuesta de 1 a 5 , sus valores de alfa de Cronbach oscilan entre $\mathrm{r}=0,89$ y 0,79 , y el Inventario de Ideación Suicida Positiva y Negativa (Positive and Negative Suicidal Ideation-PANSI), adapatación Osman y col. (33). La versión para efectos de esta investigación fue la traducida y validada 
por Villalobos-Galvis (34), la cual consta de 14 ítems, de los cuales, 6 corresponden a ideación suicida positiva (factores protectores) y 8 a ideación suicida negativa (factores de riesgo); estos ítems se evalúan indagando por las últimas dos semanas. Las opciones de respuesta varían de 0 a 4 puntos su índice de confiabilidad fue de $r=0,899$.

Finalmente se aplicó la Escala de resiliencia SV-RES de Saavedra y Villalta (35), compuesta por 60 ítems que evalúan 12 factores resultante de la visión de sí mismo, la visión del problema y la respuesta resiliente del sujeto desde las afirmaciones yo soy, yo estoy, yo tengo y yo puedo. Los factores son: Identidad (15), Autonomía (6-10), Satisfacción (11-15), Pragmatismo (16-20), Vínculos (21-25), Redes (26-30), Modelos (31-35), Metas (3640), Afectividad (41-45), Autoeficacia (46-50), Aprendizaje (51-55), Generatividad (56-60). El indicador $r=0,76$. Y la guía de entrevista, que para efectos de este estudio se elaboró un total de 33 preguntas que guiaron la recolección de datos cualitativos centrados en los factores de riesgo y protección explorando fuentes de bienestar/malestar psicológico. Estas peguntas fueron seleccionadas después de un proceso de evaluación de jueces expertos.

Desde el programa institucional de excelencia académica apoyando procesos de permanencia estudiantil se convocó a los estudiantes que hacían parte del registro de víctimas, a participar de la investigación. Una vez socializados los propósitos del estudio los estudiantes acudieron a dos sesiones voluntariamente. En la primera sesión firmaron formulario de consentimiento informado y a los menores de edad se les solicitó la autorización por escrito de los padres para participar. En estos formularios se explicaron los objetivos, procedimiento, riesgos, beneficios, voluntariedad y confidencialidad de los subproductos de esta investigación. De igual forma, una auxiliar de investigación, con el acompañamiento de una psicóloga entrenada en la aplicación de los instrumentos aplicó el cuadernillo de pruebas. En la segunda sesión, una de las investigadoras realizó las entrevistas individualmente. La duración promedio de cada entrevista fue de $2 \mathrm{~h}$.

Los datos cuantitativos fueron tratados utilizando el programa estadístico IBM SPSS versión 20 para Windows. Se consideraron variables vinculadas directamente con la salud mental en dos direcciones. Una negativa que integra: depresión, estrés percibido e ideación suicida. Y otra positiva que involucra: la satisfacción con la vida, la autoestima y el optimismo. Se utilizó estadística descriptiva para dar cuenta de las características demográficas de la muestra y los resultados de cada escala y análisis de correlaciones de Spearman considerando el tamaño de la muestra. Los datos cualitativos se analizaron con apoyo del software NVIVO utilizando técnicas de teoría fundamentada y diseño emergente, se efectuó la codificación abierta y partir de esta surgieron las categorías mediante la comparación constante (36).

\section{RESULTADOS}

El análisis descriptivo de los factores protectores y de riesgo para la salud mental de los participantes, mostró niveles de estrés percibido elevados en un segmento importante (40\%), así como la presencia de factores de riesgo para el suicidio representada en la variable ideación negativa (12\%). En esta línea, la sintomatología depresiva fue menor, aunque un $48 \%$ se ubicó en puntajes medios que refieren la presencia de síntomas para depresión sub-clínica. Los datos expuestos sugieren un menoscabo en la salud mental de los estudiantes víctimas de conflicto armado representado en sintomatología depresiva (aunque no clínica), altos niveles de estrés y la presencia de ideación vinculada al suicidio (Cuadro 1).

El análisis de los factores considerados como protectores mostró datos coherentes con lo expuesto anteriormente, puesto que se estableció un porcentaje muy bajo de participantes con altos niveles de autoestima y optimismo. Contario a esto, las puntuaciones obtenidas en satisfacción con la vida y la ideación positiva ubicaron a un porcentaje mayor de los participantes en rangos medios y altos. Es decir, a pesar de la presencia importante de factores de riesgo, bajos niveles de autoestima y optimismo, los participantes experimentan de manera importante pensamientos de protección frente al suicidio, tales como el sentido de control, la autoconfianza, las 
expectativas positivas, la alegría y la satisfacción con la vida (Cuadro 1).

Por su parte, la evaluación de la resiliencia arrojó puntajes bajos en todas las sub-escalas, así como en la puntuación global (Cuadro 2). Esto pone en evidencia una desventaja importante sobre cuatro aspectos fundamentales, la respuesta resiliente, la visión abordable del problema, la visión de sí mismo y las condiciones de base. Los cuales son recursos necesarios para superar la adversidad.

Cuadro 1

Factores protectores y de riesgo

\begin{tabular}{|c|c|c|c|c|c|c|}
\hline Variable & Mínimo & Máximo & \pm & Bajo & Medio & Alto \\
\hline Depresión & 22 & 50 & $35,56 \pm 7,26$ & $52 \%$ & $48 \%$ & $0 \%$ \\
\hline Estrés percibido & 5 & 33 & $17,36 \pm 7,37$ & $60 \%$ & $0 \%$ & $40 \%$ \\
\hline Ideación negativa & 0 & 10 & $1,08 \pm 2,61$ & $72 \%$ & $16 \%$ & $12 \%$ \\
\hline Autoestima & 16 & 35 & $27,00 \pm 3,91$ & $28 \%$ & $52 \%$ & $20 \%$ \\
\hline Optimismo & 16 & 28 & $22,36 \pm 2,73$ & $16 \%$ & $68 \%$ & $16 \%$ \\
\hline $\begin{array}{l}\text { Satisfacción } \\
\text { con la vida }\end{array}$ & 3 & 18 & $13,08 \pm 4,29$ & $32 \%$ & $4 \%$ & $64 \%$ \\
\hline Ideación positiva & 14 & 24 & $19,08 \pm 3,13$ & $0 \%$ & $0 \%$ & $100 \%$ \\
\hline
\end{tabular}

Fuente: Elaboración propia.

Cuadro 2

Resiliencia

\begin{tabular}{|c|c|c|c|c|c|c|}
\hline Variable & Mínimo & Máximo & \pm & Bajo & Medio & Alto \\
\hline Identidad & 5 & 13 & $7,16 \pm 1,97$ & $100 \%$ & $0 \%$ & $0 \%$ \\
\hline Autonomía & 5 & 17 & $8,20 \pm 2,55$ & $100 \%$ & $0 \%$ & $0 \%$ \\
\hline Satisfacción & 5 & 17 & $8,52 \pm 2,94$ & $100 \%$ & $0 \%$ & $0 \%$ \\
\hline Pragmatismo & 5 & 17 & $8,36 \pm 2,97$ & $100 \%$ & $0 \%$ & $0 \%$ \\
\hline Vínculos & 5 & 14 & $8,00 \pm 2,48$ & $100 \%$ & $0 \%$ & $0 \%$ \\
\hline Redes & 5 & 15 & $8,24 \pm 2,61$ & $100 \%$ & $0 \%$ & $0 \%$ \\
\hline Modelos & 5 & 11 & $7,64 \pm 2,05$ & $100 \%$ & $0 \%$ & $0 \%$ \\
\hline Metas & 5 & 18 & $8,00 \pm 2,81$ & $100 \%$ & $0 \%$ & $0 \%$ \\
\hline Afectividad & 5 & 16 & $9,08 \pm 2,85$ & $100 \%$ & $0 \%$ & $0 \%$ \\
\hline Autoeficacia & 5 & 12 & $8,16 \pm 2,17$ & $100 \%$ & $0 \%$ & $0 \%$ \\
\hline Autoaprendizaje & 5 & 13 & $8,28 \pm 2,35$ & $100 \%$ & $0 \%$ & $0 \%$ \\
\hline Generatividad & 5 & 16 & $7,64 \pm 2,64$ & $100 \%$ & $0 \%$ & $0 \%$ \\
\hline Global resiliencia & 61 & 158 & $97,28 \pm 21,7$ & $100 \%$ & $0 \%$ & $0 \%$ \\
\hline
\end{tabular}

Fuente: Elaboración propia. 
En total se establecieron 49 códigos, que se ubicaron en 661 referencias correspondientes a 25 entrevistas recolectadas. Se identificaron seis categorías: satisfacción, redes y conexión social, proyección, recursos personales, crecimiento y afectividad (Cuadro 3).

Redes y conexión social, se refiere a la percepción que tienen los sujetos sobre su vinculación con personas o instituciones que ofrezcan contextos de desenvolvimiento que nutran su desarrollo. Constituyéndose en la plataforma social que le da soporte al individuo para su propio crecimiento. Así, las interpretaciones de los estudiantes indagados refieren capacidad para establecer relaciones positivas, pero éstas se limitan casi exclusivamente a la familia como núcleo central y en pocos casos se hallan discursos referidos a los pares y la universidad. En este sentido, es importante señalar que los entrevistados refieren continuamente sentirse solos debido a que se encuentran lejos de su red primaria apoyo, así como también expresan con recurrencia serias y graves dificultades de relacionamiento social básico con personas ajenas a su sistema familiar.

Proyección, se configura como una categoría que contiene dos elementos; el establecimiento de metas y la percepción de control. La proyección hace referencia a la capacidad del individuo para hacer conciencia sobre sus propios recursos, y de acuerdo con ello, y con la imagen esperada de sí mismos, se establezcan logros a alcanzar de manera razonable. Esta categoría se vincula fuertemente con el proyecto de vida y en los entrevistados, su contenido se relaciona constantemente con la culminación de sus carreras universitarias. Este punto es primordial para el crecimiento personal, familiar y social, considerando que, en gran parte de los participantes, alcanzar este logro implicaría ser los primeros profesionales de sus familias y la posibilidad de movilizar positivamente los recursos hacia el mejoramiento de la calidad de su grupo primario.

Recursos personales, aluden a todos los insumos personales del sujeto para afrontar las exigencias del contexto en el que se ubican, los cuales se configuran subjetivamente para que sean desplegados cuando se requiera. En los participantes se logró identificar que cuentan con un autoconcepto positivo, capacidad de goce y actitud optimista, aunque surgen algunos rasgos resilientes, estos aún no se configuran en los sujetos.

Crecimiento, surge como una categoría que alude a la capacidad de optimizar los recursos individuales que permiten al individuo atribuir un sentido a la existencia mediante la expansión de sus fortalezas. El crecimiento denota el desarrollo personal y, además, la habilidad para autogestionar reflexivamente mediante el análisis continuo sobre las acciones propias. De esta manera, en los participantes de evidenció el crecimiento como un aspecto primordial en la vida y en menor medida apareció la capacidad de autorreflexión.

Afectividad negativa, se entiende como la orientación del individuo a experimentar sentimiento y emociones negativos que generan una marcada percepción de malestar psicológico. Las respuestas de los participantes constantemente incluyeron referencias sobre la experimentación frecuente de frustración e impulsividad, estados de irritabilidad y agresividad que limitaban la interacción con los otros.

Una vez realizado el análisis de comparación constante del cual se derivaron las categorías descritas anteriormente, se procedió a recodificar las referencias en una base de datos Excel a fin de establecer que la existencia de correlaciones entre los elementos identificados y las categorías en las que se ubicó cada uno de ellos. Los resultados mostraron correlaciones moderadas y altas entre cada uno de los elementos con su respectiva dimensión evidenciando la existencia de un modelo teórico consistente derivado del análisis ejecutado (Cuadro 3).

Seguidamente se procedió a identificar si los factores o dimensiones halladas en el análisis cualitativo guardaban relación entre ellos denotando la existencia de un constructo denominado salud mental que a su vez contenía varios aspectos. Los resultados mostraron la existencia correlaciones moderadas y altas entre todos los factores identificados para el análisis de la salud mental en víctimas (Cuadro 4).

Para establecer un modelo de salud mental de víctimas del conflicto armado, estableciendo la dirección de influencia entre las variables 


\section{ALARCÓN-VÁSQUEZ Y, ET AL}

Cuadro 3

Categorías emergentes

\begin{tabular}{|c|c|c|c|}
\hline Categoría & Elementos & $\mathbf{0}$ & Referencias \\
\hline Redes y conexión social & $\begin{array}{l}\text { Soporte social/ } \\
\text { aislamiento } \\
\text { Relaciones positivas } \\
\text { Capacidad de ayuda } \\
\text { Habilidades sociales (-) }\end{array}$ & $\begin{array}{l}0,497 * * \\
0,532 * * \\
0,502 * * \\
0,767 * *\end{array}$ & 195 \\
\hline Proyección & $\begin{array}{l}\text { Metas y compromiso } \\
\text { Baja percepción de control }\end{array}$ & $\begin{array}{l}0,731 * * \\
0,869 * *\end{array}$ & 160 \\
\hline Satisfacción & $\begin{array}{l}\text { Individual } \\
\text { Social } \\
\text { Familiar } \\
\text { Académica } \\
\text { Afectiva (-) }\end{array}$ & $\begin{array}{l}0,715 * * \\
0,753 * * \\
0,549 * * \\
0,641 * * \\
0,767 * *\end{array}$ & 127 \\
\hline Recursos personales & $\begin{array}{l}\text { Autoconcepto } \\
\text { Afrontamiento } \\
\text { Capacidad de goce } \\
\text { Optimismo }\end{array}$ & $\begin{array}{c}0,700 * * \\
0,610 * * \\
0,841 * * \\
0,944 *\end{array}$ & 123 \\
\hline Crecimiento & $\begin{array}{l}\text { Desarrollo individual } \\
\text { Autoreflexión } \\
\text { Depresión } \\
\text { Experiencia traumática }\end{array}$ & $\begin{array}{l}0,752 * * \\
0,917 * * \\
0,544 * * \\
0,468 * *\end{array}$ & 41 \\
\hline Afectividad negativa & $\begin{array}{l}\text { Frustración/impulsividad } \\
\text { Limitaciones } \\
\text { Afectación }\end{array}$ & $\begin{array}{l}0,625 * * \\
0,716 * * \\
0,718 * *\end{array}$ & 15 \\
\hline
\end{tabular}

$\mathrm{Q}=$ Rho Spearman correlación del elemento con la categoría. $* * \mathrm{P}=>0,1$

Fuente: Elaboración propia.

Cuadro 4

Correlación entre factores

\begin{tabular}{|c|c|c|c|c|c|c|}
\hline Factor & $\mathbf{1}$ & 2 & 3 & 4 & 5 & 6 \\
\hline \multicolumn{7}{|l|}{ Redes conexión social } \\
\hline Proyección & $0,565^{* *}$ & & & & & \\
\hline Satisfacción & $0,592 * *$ & & & & & \\
\hline Recursos personales & $0,465 * *$ & & $0,626 * *$ & & & \\
\hline Crecimiento & $0,426^{* *}$ & & $0,622 * *$ & $0,664 * *$ & & \\
\hline Afectividad & & & $-0,686^{* *}$ & & $-0,466^{*}$ & \\
\hline
\end{tabular}

$* * \mathrm{P}=>0,1 ; * \mathrm{P}=>0,5$

Fuente: Elaboración propia. 
identificadas en el análisis cualitativo se ejecutó un análisis de regresión múltiple. Sus resultados mostraron que para la variable proyección el coeficiente de correlación múltiple $\mathrm{r}$ ajustado $=0,540, R^{2}=0,597$, Durbin Watson 2,37 (error típico de la estimación 0,577). Estos datos sugieren que en conjunto las variables redes/ conexión social, crecimiento y recursos explican en un $59 \%$ la variable proyección (Cuadro 5).
Por otra parte, el modelo de predicción de la satisfacción arrojó un coeficiente de correlación múltiple es r ajustado $=0,660, \mathrm{R}^{2}=0,688$, Durbin Watson 1,93 (error típico de la estimación $0,939)$. Lo anterior, muestra que los recursos y la afectividad explican en un $68 \%$ las variaciones en la satisfacción (Cuadro 6).

Tabla 5

Modelo multivariante. Dependiente Proyección

\begin{tabular}{lccccc}
\hline Variable & \multicolumn{2}{c}{$\begin{array}{c}\text { Coeficiente no } \\
\text { estandarizado }\end{array}$} & $\begin{array}{c}\text { Coeficiente } \\
\text { estandarizado }\end{array}$ & T & Error \\
& B & Betándar & & P \\
\cline { 2 - 4 } & 0,859 & 0,367 & & 2,338 & 0,029 \\
Constante & 0,556 & 0,133 & 0,646 & 4,181 & 0,0001 \\
Redes conexión social & $-0,676$ & 0,198 & $-0,610$ & $-3,404$ & 0,003 \\
Crecimiento & 0,262 & 0,114 & 0,426 & 2,301 & 0,032 \\
Recursos & & & & \\
\hline
\end{tabular}

El coeficiente de correlación múltiple es r ajustado $=0,540, \mathrm{R} 2=0.597$, Durbin Watson 2,37 (error típico de la estimación 0,577).

Fuente: Elaboración propia.

Tabla 6

Modelo multivariante. Dependiente Satisfacción

\begin{tabular}{lccccc}
\hline Variable & \multicolumn{2}{c}{$\begin{array}{c}\text { Coeficiente no } \\
\text { estandarizado }\end{array}$} & $\begin{array}{c}\text { Coeficiente } \\
\text { estandarizado }\end{array}$ & T & Error \\
\cline { 2 - 4 } & B & Betándar & & \\
Constante & 2,934 & 0,561 & & 5,228 & 0,001 \\
Recursos & 0,505 & 0,146 & 0,434 & 3,463 & 0,002 \\
Afectividad & $-0,653$ & 0,140 & $-0,584$ & $-4,657$ & 0,001 \\
\hline
\end{tabular}

El coeficiente de correlación múltiple es $\mathrm{r}$ ajustado $=0,660, \mathrm{R} 2=0,688$, Durbin Watson 1,93 (error típico de la estimación $0,939)$.

Fuente: Elaboración propia.

Finalmente, se definió la propuesta de un modelo de salud mental de las víctimas del conflicto armado derivado de los análisis previos que muestra la dirección de influencia entre las dimensiones de salud mental ubicada en el discurso de los participantes mediante la aplicación de la entrevista. Como se muestra en la Figura 1, la salud mental involucra una serie dimensiones que guardan profunda relación, así surgen como categorías importantes la proyección y la satisfacción del sujeto. Las redes/conexión social y el crecimiento afectan 
y son afectados por la proyección de la persona y a su vez, la proyección influye directamente sobre los recursos del individuo, los cuales, junto con la afectividad influyen y son influidos por la satisfacción del individuo.

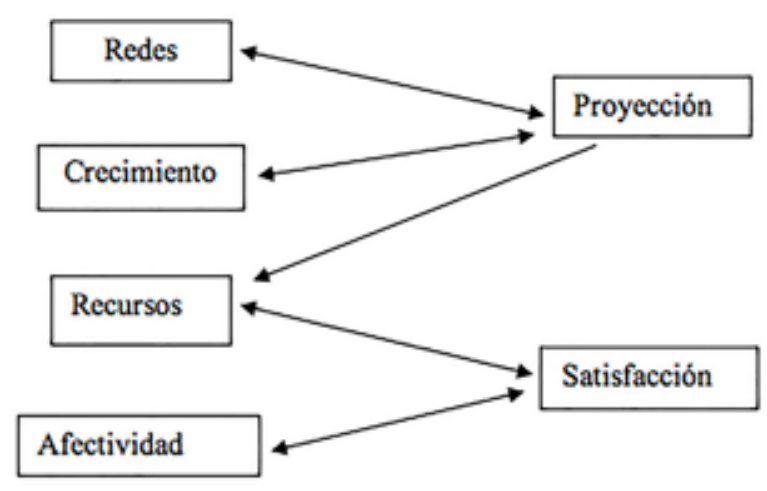

Figura 1. Modelo multivariante. Coeficientes de regresión.

\section{DISCUSIÓN}

El análisis de la salud mental en víctimas del conflicto armado constituye en un reto para múltiples esferas de la sociedad, pero de manera especial para la institucionalidad en salud y educación. El presente estudio, muestra que no existen líneas definidas para la intervención de la salud mental de los sujetos, en especial, si estos hacen parte de una institución de educación, espacio que se configura en piedra angular para jalonar múltiples capacidades.

La salud mental de los universitarios víctimas del conflicto, indudablemente es un reto para las instituciones de educación superior, máxime cuando los resultados muestran un menoscabo en las habilidades básicas para establecer relaciones sociales, datos que coinciden con los resultados de otros estudios $(14,37)$. Al respecto, diferentes investigaciones exponen la existencia de una estrecha relación entre las habilidades para interactuar con otros y el ajuste psicológico.

Uno de los aspectos en relación con la salud mental que se encontró afectado en las víctimas del conflicto armado, es el desarrollo de indicadores depresivos, coincidiendo con los hallazgos de otros estudios $(14,15)$. Por otro lado, en esta población se ve menoscabada la autoestima ya que frente a ciertos hechos violentos se pierde la valía y la dignidad en especial frente a la posibilidad de enfrentarse a la muerte, las personas se ven sometidas a depender de sus victimarios, lo cual es una situación muy difícil de enfrentar como lo plantearon otros autores $(9,10)$.

Considerando que la universidad es un contexto importante para las víctimas del conflicto, será necesario que desde la institución se establezcan mecanismos educativos que favorezcan la incorporación de esta población a las nuevas realidades que les ofrece el contexto, ampliando la percepción de la universidad como un medio que ofrece no solo apoyo académico, sino además que aporta a su desarrollo integral.

Un aspecto interesante dentro de los hallazgos fue la proyección como categoría de transformación positiva que favorece la salud mental de los sujetos. En este sentido, será necesario que la universidad tome un papel más activo frente a esta población afianzando el proyecto de vida, considerando que éste gira alrededor de la culminación de una carrera universitaria, configurándose como un aspecto central de movilidad social (38).

Por su parte, un aspecto preocupante que surgió en los participantes se vincula con la afectividad negativa, es decir, la tendencia a experimentar emociones y sentimientos negativos, que con frecuencia se refieren a ira, agresividad, frustración y afectación por la vivencia experiencias traumáticas. Estos resultados confirman lo encontrado por múltiples investigaciones que muestran graves afectaciones en la salud mental de las víctimas de violencia (39-41), y que llaman la atención a la universidad como un espacio en el que se despliegan habilidades, pero también que se hace evidente algunas limitaciones como estas, que se relacionan directamente con el desajuste psicológico. Así será necesario que instituciones de educación superior que reciben a esta población consideren la formulación de programas que promuevan el bienestar psicológico y la resiliencia en estos estudiantes, y teniendo en cuenta que el modelo derivado de este estudio mostró como elementos principales la proyección 


\section{SALUD MENTAL DE UNIVERSITARIOS VÍCTIMAS DEL CONFLICTO ARMADO}

y la satisfacción, el trabajo académico y de orientación pueden incorporar esto (40).

\section{REFERENCIAS}

1. Bravo C. Biotécia, Saúde Mental e Género. Acta Bioeth. 2006;12(2):169-175.

2. Jiménez W. Salud Mental en el posconflicto de Colombia. Re Crim. 2009;51(1):179-192.

3. Mogollón-Canal O, Villamizar-Carrillo D, PadillaSarmiento S. Salud Mental en la educación superior: una mirada desde la salud pública. Rev Ciencia Cuidado. 2016;13(1):103-120.

4. Organización Mundial de la Salud OMS. Salud Mental: un estado de bienestar. OMS; 2013.

5. Muñoz CO, Restrepo D, Cardona, D. Construcción del concepto de Salud mental positiva. Revisión sistemática. Rev Panam Salud Púb. 2016;39(3):166173.

6. Martín L, Ochotorena J. Trastorno por estrés postraumático en víctimas de situaciones traumáticas. Psicothema. 2004;16(1):45-49.

7. Campo-Arias A, Oviedo H, Herazo, E. Prevalencia de síntomas, posibles casos y trastornos mentales en víctimas del conflicto armado interno en situación desplazamiento en Colombia. Una revisión sistemática. Rev Colomb Psiquiatr. 2014;43(4):177185.

8. Castaño G, Sierra G, Sánchez D, Semenova N, Salas C, Buitrago C, et al. Salud Mental en víctimas de desplazamiento forzado por la violencia en Colombia. El caso de Bogotá,Medellín y Buenaventura. Medellín: CES;2018.

9. Ministerio de Salud y Protección Social-Colciencias. Encuesta Nacional de Salud Mental. 2015. Tomo 1. Bogotá: Ministerio de Salud y Protección Social; 2015.

10. Campo-Arias A, Herazo, E. Estigma y Salud mental en personas víctimas del conflicto armado interno colombiano en situación de desplazamiento forzado. Rev Colomb Psiquiatr. 2014;43(4):212-217.

11. Bernardini-Zambrini DA. Salud mental, salud pública y políticas públicas: parecido pero distinto forzado. Semergen. 2014;40(4):175-176.

12. World Healtht Organization. Mental health: strengthening our response. WHO; 2018.

13. Organización Mundial de la Salud. Plan de acción sobre Salud Mental 2013-2020, OMS; 2013.

14. Larizgoitia I, Izarzuaga I, Iraurgi I, Ballesteros J, Forero C, Markez I, et al. Impacto de la violencia colectiva en salud. Resultados del estudio ISAVIC en el país Vasco. Gac Sanit. 2011;25(2):108-114.
15. Steel Z, Chey T, Silove D, Mamane C, Bryant RA, Van Ommeren M. Association of torture and other potentially traumatic events with mental health outcomes among populations exposed to mass conflict and displacement: A systematic review and metaanalysis. JAMA. 2009;302(5):537-549.

17. Bell V, Mendez F, Martinez C, Palma P, Bosch, M. Characteristics of the Colombian armed conflict and the mental health of civilians living in active conflict zones. Conflict and Health, 2012;6(1):1-8.

18. Londoño A, Romero P, Casas G. The association between armed conflict, violence and mental health: a cross sectional study comparing two populations in Cundinamarca department, Colombia. Conflict and Health, 2012;6(12):1-6.

19. Arévalo L. Atención y reparación psicosocial en contextos de violencia sociopolítica: una mirada reflexiva. Revista de Estudios Sociales. 2010;36:2939.

20. Ministerio del Interior. Ley 1448 de 10 de Junio de 2011. Ley de restitución de tierras y decretos reglamentarios. Bogotá: Ministerio del Interior; 2012.

21. MorenoA,Díaz M. Posturas en la atención psicosocial a víctimas del conflicto armado en Colombia. AGO. USB. 2016;43(4):198-213.

22. Huerta J. El rol de la educación en la movilidad social de México y Chile ¿La desigualdad por otras vías? RMIE. 2012;17(52):65-88.

23. Sanhueza F, Cornejo P, Leyton J. La educación como agente de movilidad social. Convergencia Educativa, 2015;(6):95-106.

24. Omwuegbuzie A, Johnson R. The Validity issue in mixed research. Research in the Schools. 2006;13(1):48-63.

25. Zung A. Self-reportdepressionscale. Arch Gen Psychiatry. 1965;12:63-70.

26. Lezama S. Propiedades psicométricas de la escala Zung para síntomas depresivos en población adolescente escolarizada colombiana. Psychol Av Discip. 1965;6(1):91-101.

27. Ceballos-Ospino G, Paba-Barbosa C, Suescún J, Oviedo H, Herazo E, Campo-Arias A. Validez y dimensionalidad de la escala de autoestima de Rosenberg en estudiantes universitarios. Pensamiento Psicológico. 2017;15(2):29-39.

28. Scheier MF, Carver CS, Bridges MV. Distinguishing optimism from neuroticism (and trait anxiety, selfmastery, and self-esteem)- a reevaluation of the Life Orientation Test. J Pers Soc Psychol. 1994;67(6):10631028.

29. Velasco M, Londoño C, Alejo I. Validación del cuestionario de optimismo disposicional usando la teoría de respuesta al ítem. Divers Perspec Psicol. 2014;10(2):275-292. 


\section{ALARCÓN-VÁSQUEZ Y, ET AL}

30. Cohen S, Kamarck T, Mermelstein R. A global meausre of perceived stress. J Health Social Behav. 1983;24(4):385-396.

31. Diener E, Emmons R, Larsen R, Giffin S. The Satisfaction with Life Scale. J Pers Assess. 1985;49:71-75.

32. Atienza F, Pons D, Balaguer I, García-Merita M. Propiedades psicométricas de la Escala de Satisfacción con la vida en adolescentes. Psicothema. 2000;12(2):331-336.

33. Osman A, Gutiérrez P, Kopper B, Barrios F, Chiros C. The positive and negative suicide ideationinventoy: Development and validation. Psychol Rep. 1998;82:783-793.

34. Villalobos-Galvis F. Validez y fiabilidad del Inventario de Ideación Suicida Positiva y Negativa -PANSI, en estudiantes colombianos. Univ Pyschol. 2009;9(2):509-520,

35. SaavedraE, Villalta M. Escala de resieliencia SV-RES para jóvenes y adultos. Chile: Ceanim; 2008.

36. Hernández-Sampieri R, Fernández C, Baptista P. Metodología de la investigación. México: Mc Graw Hill; 2010.
37. Charry-Lozano L. Impactos psicológicos y psicosociales en víctimas sobrevivientes de masacre selectiva en el marco del conflicto en el suroccidente colombiano en el año 2011. Colombia Forense. 2016;3(2):51-60.

38. Vélez Vásquez M. Educación Universitaria como factor de movilidad social. Impactos psicológicos y psicosociales en víctimas sobrevivientes de masacre selectiva en el marco del conflicto en el suroccidente colombiano en el año 2011. Rev Estud Interdis Cienc Soc. 2014;16(2):207-225.

39. Gómez-Restrepo C, Tamayo-Martínez N, Buitrago G, Guarnizo-Herreno C, Garzón Orjuerla N, EslavaSchmalbach J, et al. Violencia por conflicto armado y prevalencia de trastornos del afecto, ansiedad y problemas mentales en la población adulta colombiana. Rev. Colomb Psiquiatr. 2016;45(s1):147-153.

40. Sheikh T, Abdulaziz M, Agunbiad S, Joseph I, Ebiti B, Adekeye O. Send to correlates of depression among internally displaced persons after post-election violence in Kaduna, North Western Nigeria. J Afecct Disord. 2015;1(170):46-51.

41. Campo-Arias A, Ospino A, Sanabria A, Guerra V, Caamaño B, Herazo E. Frecuencia y variables asociadas a estigma-discriminación percibido en víctimas del conflicto armado colombiano. Cad Saúde Púb. 2017;33(11):1-6. 